https://creativecommons.org/licenses/by/4.0/

\title{
ESTANDARIZACIÓN DEL PROCESO DE CONSUMIBLES EN EL SENADO DE LA REPÚBLICA DE COLOMBIA ${ }^{1}$
}

\author{
Standardization of the consumables process \\ in the Senado de la República of Colombia
}

LORENA VANESSA CARVAJAL ESCOBAR ${ }^{2}$, JUAN FELIPE VALDERRAMA PÉREZ ${ }^{3}$, EVER ÁNGEL FUENTES ROJAS, MBA

Recibido:4 de diciembre de 2020. Aceptado:15 de enero de 2021

DOI: http://dx.doi.org/10.21017/rimci.2021.v8.n15.a92

\begin{abstract}
Resumen
Las entidades estatales de un país tienen la responsabilidad de aplicar y fomentar en su territorio, el cumplimiento de los Objetivos de Desarrollo Sostenible propuestos por la Organización de Naciones Unidas. Dentro de estos, se encuentra el "garantizar modalidades de consumo y producción sostenibles"[1]; una de sus metas es que, a 2030, se debe "reducir considerablemente la generación de desechos mediante actividades de prevención, reducción, reciclado y reutilización". Para el caso del Senado de la República de Colombia, interesada en cumplir con dichos objetivos, se presenta una metodología acorde para la estandarización del proceso de consumibles (residuos asociados a productos de oficina como el papel, plástico, cartón, mezcladores, vasos, etc.), el cual tiene un papel importante al interior de la institución en relación con la optimización de recursos. El método aplicado se basó en herramientas de ingeniería que permitieron analizar y evaluar los procedimientos que tienen mayor influencia, lo cual tuvo como resultado la realización de toda la documentación del proceso, con sus respectivos tiempos de ejecución, indicadores y evaluación de impacto. La aplicación de dichos lineamientos permitirá tener un mayor control sobre el manejo y disposición final de los productos remanentes, lo cual constituye un avance y desarrollo en el aspecto procedimental y ambiental orientado a ser una organización sostenible.
\end{abstract}

Palabras clave. Estandarización de procesos; residuos; consumibles; Senado de la República de Colombia; entidad estatal; Rama Legislativa.

\begin{abstract}
The state entities of a country have the responsibility of applying and promoting in their territory, the fulfillment of the Sustainable Development Goals proposed by the United Nations. Among these, is the "guaranteeing sustainable consumption and production modalities" [1]; one of its goals is that, by 2030, the generation of waste should be "considerably reduced through prevention, reduction, recycling and reuse activities". In the case of the Senate of the Republic of Colombia, interested in meeting these objectives, a consistent methodology is presented for the standardization of the consumables process (waste associated with office products such as paper, plastic, cardboard, mixers, glasses, etc.), which has an important role within the institution in relation to the optimization of resources. The applied method was based on engineering tools that allowed the analysis and evaluation of the procedures that have the
\end{abstract}

1 Artículo producto del proyecto investigativo de la estandarización de procesos en la División de Bienes y Servicios del área administrativa del Senado de la República, ejecutado en el año 2019 y 2020 desde el programa de ingeniería de la Universidad Libre, sede Bogotá

2 Estudiante de ingeniería industrial. Universidad Libre, Colombia. Correo electrónico: lorena.carvajal@unilibre.edu.co ORCID: https:// orcid.org/0000-0002-7659-3194

3 Estudiante de ingeniería industrial. Universidad Libre, Colombia. Correo electrónico: juan.valderrama@unilibre.edu.co ORCID: https:// orcid.org/0000-0003-3298-9119

4 MBA, Ingeniero industrial. Universidad Libre, Colombia. Docente de jornada completa del programa de ingeniería industrial. Universidad Libre, Colombia. Correo electrónico: ever.fuentes@unilibre.edu.co ORCID: https://orcid.org/0000-0001-9671-5884 
greatest influence, which resulted in the completion of all the documentation of the process, with their respective execution times, indicators and impact evaluation. The application of these guidelines will allow greater control over the management and final disposal of the remaining products, which constitutes progress and development in the procedural and environmental aspects aimed at being a sustainable organization.

Keywords-Standardization of processes; waste; consumables; Senate of the Republic of Colombia; state entity; Legislative branch.

\section{INTRODUCCIÓN}

L OS RESIDUOS sólidos son el subproducto de la actividad del hombre y se han producido desde los inicios de la humanidad. Cada día aumentan en cantidad y variedad como consecuencia del incremento de la población humana y del desarrollo tecnológico e industrial. Su disposición final incorrecta ha ocasionado grandes problemas al ambiente, contaminando agua, aire y suelo [2]. Algunos de los desechos más utilizados en las organizaciones son los ordinarios (de productos consumibles), generados en el desempeño normal de actividades de oficina, áreas comunes y cafeterías, sin embargo, a pesar de que se encuentran en la mayoría de las organizaciones, no siempre se hace un uso adecuado de los mismos.

Estos residuos toman gran importancia en el mundo, y por ello han sido objeto de estudio a nivel nacional e internacional, en investigaciones y proyectos asociados a su manejo y disposición para obtener resultados favorables en distintos aspectos. Un ejemplo de estos, es el trabajo hecho por Jiménez Coronado, Ferreira Simmonds y Fernández Villareal [3]; el cual tiene como objetivo diagnosticar el estado ecológico de los procesos que hacen parte de una organización, identificando sus aspectos y valorando los impactos que se puedan generar en su ejecución. La metodología se basó en tres etapas que fueron: Identificar los procedimientos más importantes mediante las visitas de los investigadores al lugar de estudio; caracterización, que permitió determinar las entradas y salidas de cada una de las actividades llevadas a cabo en la identificación, y una matriz de impactos en el medio ambiente de acuerdo con los procedimientos. Esto permitió, en el estudio de Jiménez Coronado y otros, valorar la práctica de la gestión ambiental en la institución, debido a que se requerían mayores esfuerzos en la planificación, que en ese momento era incipiente, por ello se concluyó que es necesario lograr mayores estrategias en la planeación y se dieron algunas sugerencias desde diferentes perspectivas para identificar adecuada- mente en qué procesos existen oportunidades de mejora y cuáles están generando cuellos de botella o reprocesos.

\section{Problemática de la institución}

El trabajo se realizó en el Senado de la República de Colombia, que, además de sus funciones legislativas, esta institución cumple también con funciones administrativas, lo cual se describe en la Ley $5^{\text {a }}$ de 1992. Los servicios administrativos y técnicos del Senado comprenden Áreas legislativa (Mesa Directiva de la Entidad, Secretario General del Senado) y administrativa (Dirección General Administrativa del Senado, que tiene como órgano superior la Comisión de Administración). Dentro de la Dirección General Administrativa se encuentran 5 Divisiones: Jurídica, de Planeación y Sistemas, de Recursos Humanos, Financiera y Presupuesto, y, de Bienes y Servicios. Es sobre esta última que se realiza el trabajo del presente artículo. La División de Bienes y Servicios tiene entre sus funciones evitar el deterioro de las instalaciones físicas; coordina el mantenimiento y reparación de equipos, muebles y edificios, entre otros. De igual forma, tramita el suministro de elementos de consumo del quehacer cotidiano, en conjunto con la Sección de Suministros, Unidades de Almacén, Correspondencia, Fotocopiado y Archivo Administrativo.

Por lo general, las entidades del Estado emplean dinero público y propenden por invertir adecuadamente este dinero, sin embargo, hay ocasiones en que las actividades que se realizan generan pérdidas por el uso inadecuado de algunos bienes y servicios que se manejan o por las diligencias que implican reprocesos y acciones innecesarias, que en muchos casos se convierte tan frecuente, que no se logra distinguir una solución de mejora en la forma de hacer las cosas. Dentro de los esfuerzos que ha realizado en el último tiempo el Senado de la República, representada por la División de Bienes y Servicios, se evidencia una oportunidad de mejora en la manera de llevar a cabo sus procesos asociados al manejo y disposición final de los recursos. 
El análisis de mejora en esta zona de la institución se convierte en un proyecto innovador en el Senado de la República, en virtud de que el proceso de consumibles actúa de manera prácticamente informal y no se tiene un control riguroso sobre los desperdicios o cualquier tipo de actividad que no genere valor en esta área y que, por el contrario, pueda llegar a ocasionar pérdidas. $\mathrm{Al}$ interior de la organización, se cuenta con formatos para el registro de algunos residuos, pero no está documentado. A lo largo de los años, la División de Bienes y Servicios ha realizado algunos programas que aportan a este procedimiento, sin embargo, no han tenido el impacto necesario para mejorarlo de forma considerable.

La División tiene entre sus objetivos poder estandarizar el proceso de consumibles, lo que permitirá dar una utilización adecuada a los residuos, materiales, etc., y su respectivo ordenamiento para eliminar así las actividades que no generan valor, a la vez que se logra impactar positivamente en el ambiente. Gracias a esto, se podrán utilizar mejor los recursos, evitar el desperdicio y fomentar el desarrollo de los procesos a nivel interno.

Se puede identificar entonces que el problema del proceso de consumibles dentro del Senado se basa en la falta de estandarización y compromiso frente al adecuado uso, orden y finalidad de estos materiales. De acuerdo con esto, es conveniente preguntarse: ¿Cómo eliminar las actividades que no representan valor en el proceso de consumibles en el Senado de la República?

\section{Proceso metodológico}

La estandarización tiene como finalidad unificar los procesos de una empresa para así controlar mejor las actividades, de una forma en el que no se desperdicie tiempo, material o recurso humano. Se puede definir a la estandarización como todo aquello que está documentado y norma el "quehacer" y el comportamiento de la gente [4].

El objetivo principal de este trabajo es estandarizar el proceso de consumibles en el Senado de la República para la eliminación de actividades que no representan valor, para el cual se formulan los siguientes objetivos específicos:
- Diagnosticar la situación actual de los procesos de consumibles para el Senado de la República

- Documentar los procesos de consumibles que se llevan a cabo para la validación de las operaciones que los integran

- Desarrollar un estudio de tiempos y movimientos de las operaciones en los procesos de consumibles para el mejoramiento en la prestación del servicio

- Establecer indicadores de los procesos de consumibles que permitan la medición de los resultados obtenidos

- Realizar el análisis de impactos en los procesos de consumibles para la determinación de la viabilidad del proyecto

El trabajo se realiza con un tipo de investigación mixta, debido a que se utilizan variables y técnicas de carácter cualitativo y cuantitativo, algunos autores la llaman investigación cuanticualitativa, y ésta se basa en la generación de ideas que lleven a una realidad intersubjetiva (combinación de las realidades objetiva y subjetiva). Esto permite dar una mirada más amplia a la investigación, a la misma vez que se da mayor confiabilidad al trabajo. La meta de la investigación mixta no es reemplazar a la investigación cuantitativa ni a la investigación cualitativa, sino utilizar las fortalezas de ambos tipos de indagación, combinándolas y tratando de minimizar sus debilidades potenciales [5].

El proceso metodológico está enfocado de la siguiente forma: como primer paso, se procederá a diagnosticar y recoger información que pueda aportar en la investigación, esto con el fin de conocer el presente escenario del proceso de consumibles, y así mismo tomar información para continuar con el siguiente paso, que consta de la ejecución de algunas entrevistas y encuestas para posteriormente aplicar algunas herramientas de ingeniería con el fin de realizar un análisis detallado de los aspectos internos y externos que favorezcan o inhiben el buen funcionamiento de la institución. Cada uno de los procedimientos que se ejecutará, debe documentarse en función de su naturaleza y de los requisitos que se tengan estipulados, esto permite dar paso a la realización de 
un estudio de tiempos, caracterizado por manejarse en actividades no rutinarias. Posteriormente, se construyen los indicadores y evaluación de impacto para obtener los resultados del trabajo.

\section{Desarrollo}

El análisis se inició con un diagnóstico de la situación actual del proceso, mediante la realización de encuestas al interior de cada una de las sedes del Senado de la República (la cual se encuentra ubicada en Bogotá, Colombia, y cuenta con siete sedes).

Para definir la cantidad de encuestas a hacer, se realizó una muestra estadística mediante el método de muestreo aleatorio simple bietápico estratificado para población conocida [6]

La representatividad de una muestra permite extrapolar y por ende generalizar los resultados observados en ésta. Por ello, será representativa o no; sólo si fue seleccionada al azar, es decir, que todos los sujetos tuvieron la misma posibilidad de ser seleccionados, $\mathrm{y}$, por otro lado, que el número de sujetos elegidos representen numéricamente a la población que le dio origen respecto del cálculo del tamaño de la muestra [7]. En la técnica del Muestreo Aleatorio Simple, cada elemento tiene una oportunidad igual e independiente de ser seleccionado, para lo cual se le asigna un número. La muestra se determina con tablas de números aleatorios [8]. La técnica de muestreo probabilístico estratificado es aquella donde se divide la población en subgrupos o estrato, de estos grupos o estratos se selecciona una muestra aleatoria, tomando al menos un elemento de cada grupo o estrato [9].

Para la realización de la muestra, se utilizó la siguiente fórmula:

$$
n=\frac{Z^{2} P Q N}{E^{2}(N-1)+Z^{2} P Q}
$$

Donde:

$\mathrm{Z}=$ desviación normal

$\mathrm{P}=$ proporción de éxito

$\mathrm{Q}=$ proporción de fracaso

$\mathrm{N}$ = tamaño de la población

$\mathrm{E}=$ error muestral
Posteriormente, se realizaron las encuestas para cada sede; los resultados se analizaron mediante un Diagrama de Paretto y se formularon las matrices estratégicas DOFA, PEYEA y PESTEL.

El Diagrama de Paretto es una herramienta que puede aplicarse para identificar las causas que ocasionan los efectos no deseados, así como también para los sucesos o fenómenos que aparecen en el ámbito empresarial, lo cual la hace muy versátil para lograr la calidad de los productos [10].

El análisis DOFA (también conocida como FODA, MAFE en español y SWOT en inglés) es una de las técnicas más empleadas en la planeación estratégica, en especial para la determinación de la posición estratégica de la organización. Por lo demás, es una importante herramienta de apoyo para la toma de decisiones generalmente usada para analizar sistemáticamente los ambientes interno y externo [11].

La matriz PEYEA (por sus siglas, Posición Estratégica y Evaluación de la Acción) es una herramienta que ayuda a encontrar la posición relativa de la empresa en el mercado tomando como referencia el diagnóstico DOFA, sirve para encontrar el enfoque de la estrategia corporativa [12]. De acuerdo con Alonso G, en 2016, el análisis PEST (también conocido como PESTEL) es una herramienta que permite identificar los factores del entorno general que afectan a la organización y al resto de agentes socioeconómicos [13]. Se trata de una herramienta que posibilita comprender la situación de la organización y demás agentes socioeconómicos para así plantear adecuadamente el presente y construir un mejor futuro para una organización.

Teniendo en cuenta todo el diagnóstico, se procedió a realizar la documentación para todo el proceso de consumibles. Documentar es definir ampliamente las responsabilidades, el lugar, el momento y la forma como debe ejecutarse cualquier actividad, pero en un sentido más amplio. Cualquier sistema implantado en la organización debe estar organizado de forma similar, particularmente cuando requiere que esas actividades se repitan de la mejor manera. De esta forma se aportan soluciones adecuadas en el momento oportuno, con el fin de evitar la proliferación de métodos y procesos, y la toma de decisiones incoherentes o complejas [14]. 
ICONTEC, indica que, en un sistema de gestión, la organización debe mantener y conservar la información documentada para apoyar la operación de sus procesos y para tener la confianza de estos se realizan según lo planificado [15].

Para este caso, se dividieron las actividades en procedimientos propuestos, que son los siguientes:

1. Manejo de área de almacenamiento

2. Distribución de productos

3. Campañas y capacitaciones

4. Clasificación de residuos

Para iniciar con la documentación, se caracterizaron dichos procesos. La caracterización es una herramienta utilizada para describir cómo funciona un proceso, en consecuencia, la planificación táctica corresponde al nivel estructural en la gestión de la calidad, permite definir la secuencia o el orden para efectuar una buena construcción de la técnica y de este modo dar cumplimiento a los requisitos de la norma [14]. De igual manera, se creó una matriz de riesgos con todas las actividades que allí se encuentran, basada en la "Guía para la identificación de los peligros y la valoración de los riesgos en seguridad y salud ocupacional (GTC 45)" de ICONTEC [16].

Seguidamente, se realizó para cada procedimiento un protocolo, que contiene distintos formatos para documentar y llevar a cabo adecuadamente los procesos, con sus respectivos diagramas de flujo. La División de Bienes y Servicios del Senado ya contaba con algunos formatos, que se relacionaron con la caracterización mencionada anteriormente. También se propusieron mejoras a algunos de los formatos ya existentes. Posteriormente, se planteó el estudio de tiempos para cada uno de los procedimientos. Debido a que la implementación tiene que pasar por aprobaciones de diferente índole para poder ponerla en marcha, no se pueden tomar todos los tiempos de las actividades, por ende, se otorgaron unas recomendaciones de tiempos basadas en el método de tiempos predeterminados (MTM), se desarrolla a partir de los valores asignados a las operaciones corporales (micro movimientos) que el analista selecciona de un manual después de analizar el método y los suma para obtener la duración necesaria para efectuar un determinado trabajo [16]. Para este caso, se acudió a tres expertos en procesos similares al de consumibles, para que pudieran dar su estimación aproximada acerca de cada una de las actividades, después, se promediaron los tiempos para así obtener la cantidad (en minutos) adecuada para realizarlas.

Con el fin de llevar a cabo un seguimiento y trazabilidad, se plantearon unos indicadores de gestión para medir el peso total de residuos sólidos; determinar la cantidad mensual de residuos sólidos ingresados en el área de almacenamiento de estos, al interior del Senado, por cada persona; medir el grado de satisfacción de los funcionarios del Senado en relación con las soluciones propuestas; determinar el cumplimiento del pedido recibido $y$, determinar la calidad de la recepción de materiales con base en lo que se pidió.

Un indicador es una magnitud que expresa el comportamiento o desempeño de un proceso, que al compararse con algún nivel de referencia permite detectar desviaciones positivas o negativas. También es la conexión entre dos medidas relacionadas entre sí, que muestra la proporción de la una con la otra [17][18].

Como paso final, y con el fin de evaluar el impacto, se realizó la Matriz de Marco Lógico propuesta por la CEPAL, y básicamente en ella se cruzan cuatro niveles de objetivos con cuatro ámbitos de asociados a la gestión, para presentar en forma muy sintética la esencia de la transformación que se espera lograr con el programa o proyecto, más los elementos del ambiente de confianza y credibilidad que debe generar la gestión [19].

\section{Desarrollo}

\section{Diagnóstico}

Como parte inicial del diagnóstico, se realizaron visitas a las diferentes sedes del Senado de la República, para poder evaluar las condiciones de cada sitio y poder así identificar los problemas que presentan. Al mismo tiempo, se tomaron datos sobre la cantidad de personas que trabajan en cada sede (esta información fue obtenida durante el último semestre de 2019), como se evidencia en la Tabla 1. 
Tabla I. Cantidad de funcionarios del Senado de la República

\begin{tabular}{|c|c|c|}
\hline Trabajadores en planta & UTL & TOTAL \\
\hline 296 & 1,000 & 1,296 \\
\hline
\end{tabular}

Fuente: Los autores, 2020

Los trabajadores en planta son los que tienen funciones netamente en el área administrativa, la UTL (Unidad de Trabajo Legislativo), se conforma de personas que trabajan directamente con los Senadores de la República. Los datos fueron suministrados por personal de la entidad.

Para realizar la muestra poblacional (la cual permite hallar el valor que se utilizará para la realización de encuestas), se tomaron los siguientes valores previos:

- Error de confianza: 95\%

- Error de muestreo: 6\%

- Proporción de éxito: 0,5

- Proporción de fracaso: 0,5

- Tamaño de población: 1,296

- Desviación normal: 1,64

Lo que arrojó como resultado una muestra de 165 trabajadores a encuestar, que posteriormente se distribuyeron entre las 7 sedes, de la siguiente manera: cada sede tiene una cantidad de residuos generados por año, cuyos valores fueron suministrados por el Senado (en datos promedios) para el presente estudio, para lo cual se halló el porcentaje de participación de cada uno, esto con el fin de distribuir el valor de la muestra poblacional en las sedes con la misma proporción que tienen los residuos en cada una, como se puede ver en la Tabla 2.

Para cada sede, se tomaron el número de encuestas que se encuentra en el valor de la columna de "muestra poblacional". Para ello, se generó una ficha técnica de la encuesta y su respectivo formato para la realización de la misma.

Las encuestas fueron hechas en un lapso de 30 días, en estas se indagaron temas asociados al funcionamiento del proceso de consumibles y al manejo, clasificación y disposición de los residuos. De igual forma, se diseñó y posteriormente se diligenció una lista de chequeo que permitió conocer en qué aspectos tenía un mayor o menor orden. Finalmente, los resultados se tabularon y con ello se realizó el Diagrama de Paretto, como se puede evidenciar en la Figura 1.

Se pudo evidenciar que aproximadamente el $80 \%$ de los problemas (o la percepción de los mismos) es causada por el $20 \%$ de las causas, que en este caso están relacionados con la falta de "Cultura y Educación" por parte de la gente, y de "No separar adecuadamente los residuos" en sus respectivas canecas. La mayoría de las personas encuestadas identificaron estos problemas.

Luego de hacer el Diagrama de Paretto, se procedió con la realización de las matrices PEYEA y

Tabla II. Cantidad de trabajadores en cada sede

\begin{tabular}{|c|c|c|c|c|}
\hline Sede & $\begin{array}{c}\text { Residuos en } \\
\text { Kilogramos }\end{array}$ & Participación \% & Población & $\begin{array}{c}\text { Muestra } \\
\text { poblacional }\end{array}$ \\
\hline 1 & 1,202 & 4 & 52 & 7 \\
\hline 2 & 1,555 & 5 & 65 & 8 \\
\hline 3 & 1,804 & 6 & 78 & 10 \\
\hline 4 & 2,004 & 6 & 156 & 10 \\
\hline 5 & 3,804 & 12 & 415 & 53 \\
\hline 6 & 10,923 & 32 & 454 & 58 \\
\hline 7 & 11,472 & 35 & 1,296 & 165 \\
\hline
\end{tabular}

Rev. Ingeniería, Matemáticas y Ciencias de la Información Vol. 8 / Núm. 15 / enero - junio de 2021; pág. 55-66 


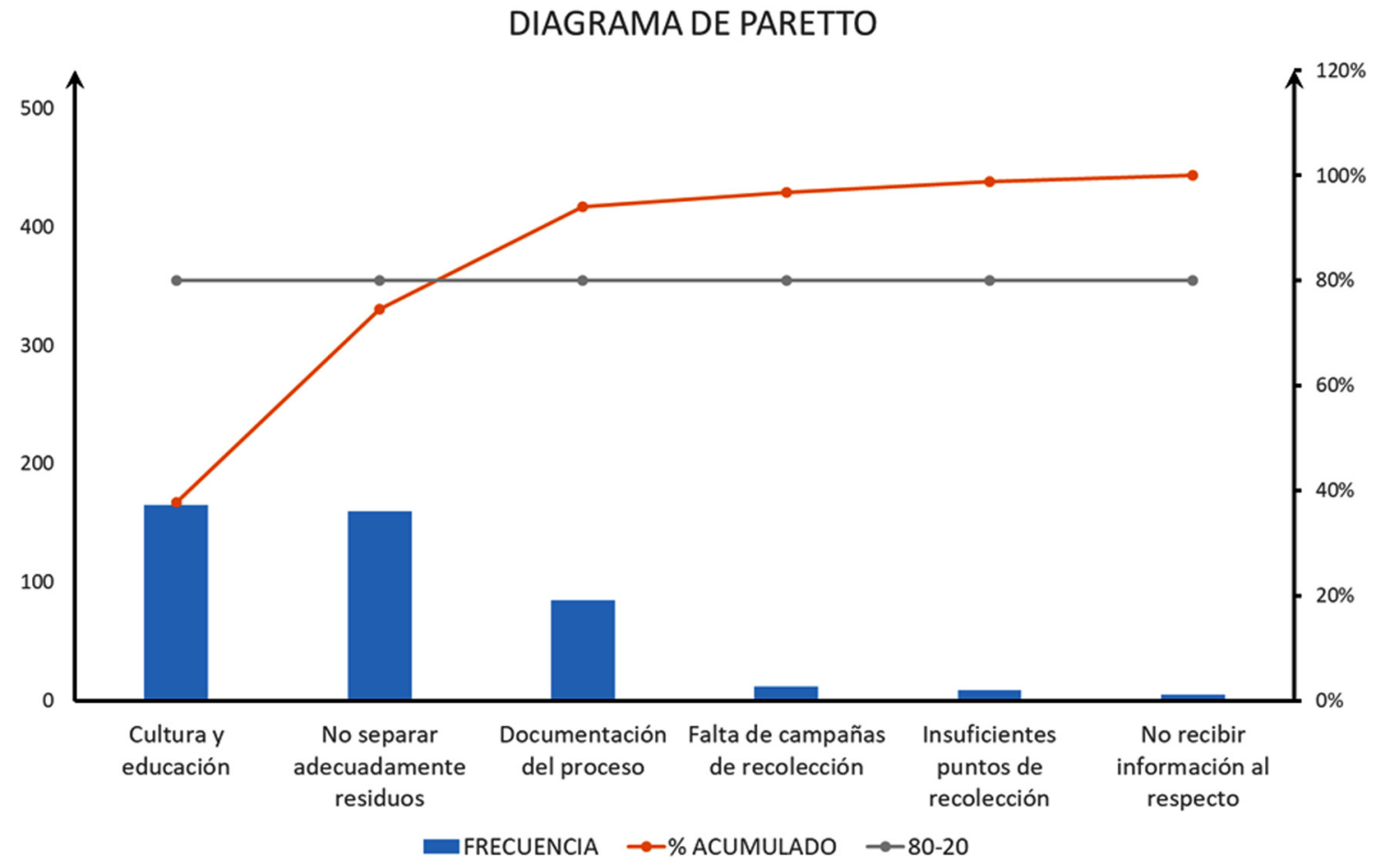

Fig. 1. Diagrama de Paretto. Fuente: Los Autores, 2020.

PESTEL. En el caso de la primera, se ponderaron diversos factores asociados a la ventaja competitiva, estabilidad ambiental y fuerzas financiera e industrial. Lo cual permitió identificar qué tipo de estrategias (conservadora, agresiva, defensiva o competitiva) conviene desarrollar al interior de la división. El resultado apunta a que se deben implementar estrategias competitivas y de estabilidad ambiental, con miras hacia la fortaleza de la entidad.

Por otro lado, para el caso de la segunda matriz (PESTEL), se hizo una ponderación también, pero basada en aspectos relacionados a los factores político, económico, social, tecnológico y económico, y fueron hechas de acuerdo con las investigaciones realizadas en las visitas. El resultado obtenido indica que el mayor impacto en la división está encaminado en los factores ecológico y social, lo cual tiene relación, principalmente en el aspecto social, ya que se está trabajando en una entidad pública que ejerce funciones constitucionales y legales (para promover el bien común y el desarrollo de la sociedad, sin ánimo de lucro), sin embargo, la división sobre la cual se efectúa el estudio, específicamente en el proceso de consumibles, las labores son netamente administrativas e independientes de las funciones legislativas que manejan otras áreas del Senado de la República.

Para finalizar con el diagnóstico y, tomando como referencia las herramientas mencionadas anteriormente, se sintetizó la información y se analizó mediante una matriz DOFA, a la misma vez que se generaron estrategias para cada uno de los factores (Debilidades, Oportunidades, Fortalezas y Amenazas).

\section{Documentación}

Continuando con la documentación, se inició generando una caracterización de los procesos basado en el Ciclo PHVA, el cual, según ICONTEC [15] permite a una organización asegurarse de que sus procesos cuenten con recursos y se gestionen adecuadamente, y que las oportunidades de mejora se determinen y se actúe en consecuencia. Este ciclo emplea el Planear, Hacer, Verificar y Actuar, que para este trabajo se realizó con las actividades de la división asociada a estos cuatro frentes. De igual forma, se especificaron unas entradas, salidas y responsables para cada una. 
Al interior de este formato, se encuentra referenciada la matriz de riesgos del proceso de consumibles, realizada principalmente para determinar los riesgos y controles pertinentes para la seguridad y salud de las personas que trabajan en el proceso de consumibles, y es aplicable a los trabajadores desde la identificación del riesgo en compra, distribución y almacenamiento, hasta el uso y disposición final de los residuos. Cuenta con la identificación de los cargos en la División de Bienes y Servicios, el tipo de actividad (rutinaria o no), la descripción y clasificación del peligro, los posibles efectos sobre la salud, unos controles en la fuente, medio e individuo, y unas ponderaciones para evaluar y valorar el riesgo, especificando también las medidas de intervención adecuadas para mitigarlo y prevenirlo.

Posteriormente, se realizó una división del proceso general en 4 procedimientos específicos que son: Manejo del área de almacenamiento, distribución de productos, campañas y capacitaciones, y clasificación de residuos. La razón de dividirlos de esa forma se fundamenta en los hitos del proceso de consumibles. Para cada apartado se efectuó un protocolo (el cual no se tiene documentado en el Senado actualmente) que contiene los diferentes formatos, manuales, instructivos y diagramas para llevar a cabo adecuadamente las actividades que cada uno contiene. Los procedimientos se formularon de la siguiente manera:

- Manejo del área de almacenamiento: establece los parámetros para su adecuado manejo en los productos consumibles. Su alcance va desde el pedido de materiales y productos hasta su disposición en el lugar final

- Distribución de productos: determina las actividades para la adecuada distribución de los productos consumibles a las diferentes sedes del Senado de la República. Tiene su alcance desde el alistamiento de los pedidos hasta su entrega en cada sede

- Campañas y capacitaciones: determina las actividades para su adecuada ejecución, asociadas al manejo de residuos en el proceso de consumibles en las diferentes sedes del Senado. Va desde la planeación de las campañas y capacitaciones hasta la retroalimentación de las mismas

- Clasificación de residuos: establece una adecuada clasificación de productos remanentes consumibles al interior de la institución para un mejoramiento en el reciclaje que se lleva a cabo. Su alcance parte de la correcta separación en las canecas establecidas, hasta su disposición final

\section{Estudio de tiempos}

Para este estudio se generaron, como paso inicial, los diagramas de procesos que contienen todas las actividades que lleva cada procedimiento, junto con la duración de cada una. La metodología aplicada fue la de Methods Time Measurement para actividades no rutinarias, cuya metodología consistió en acudir a tres expertos (dos de ellos en temas y procesos afine, y uno que trabaja directamente en el Senado de la República), para que pudieran dar sus estimaciones, teniendo en cuenta cada diligencia, las distancias y la cantidad de material a distribuir de un lugar a otro, basados en las condiciones generales estipuladas en los protocolos propuestos anteriormente. Al final, se promediaron las tres estimaciones, lo que arrojó los tiempos promedios (en minutos) para cada actividad. Tabla III.

Como se puede evidenciar, hay un total de 55 actividades en todo el proceso, con un tiempo aproximado de 2.942 minutos para hacerlo de for-

Tabla III. Datos por cada procedimiento

\begin{tabular}{|c|c|c|c|c|c|c|c|c|}
\cline { 2 - 9 } \multicolumn{1}{c|}{} & \multicolumn{2}{c|}{\begin{tabular}{c} 
Procedimiento \\
\multicolumn{1}{c|}{}
\end{tabular}} & \multicolumn{2}{c|}{$\begin{array}{c}\text { Procedimiento } \\
\mathbf{1}\end{array}$} & \multicolumn{2}{c|}{ Procedimiento } & \multicolumn{2}{c|}{ Procedimiento } \\
\hline TA & $\mathbf{C}$ & $\mathbf{T}$ & $\mathbf{C}$ & $\mathbf{T}$ & $\mathbf{C}$ & $\mathbf{T}$ & $\mathbf{C}$ & $\mathbf{T}$ \\
\hline Op & 15 & 357 & 6 & 188 & 18 & 1163 & 10 & 890 \\
\hline Insp & 2 & 153 & 3 & 44 & 0 & 0 & 1 & 147 \\
\hline Mix & 0 & 0 & 0 & 0 & 0 & 0 & 0 & 0 \\
\hline Tot & 17 & 510 & 9 & 232 & 18 & 1163 & 11 & 1037 \\
\hline
\end{tabular}

Rev. Ingeniería, Matemáticas y Ciencias de la Información Vol. 8 / Núm. 15 / enero - junio de 2021; pág. 55-66 
ma completa. Sin embargo, no todo se hace de forma simultánea o continua; algunas no son dependientes de otras, por ello el tiempo puede variar en el momento en que se realicen.

\section{Indicadores}

Los indicadores de gestión fueron realizados para evaluar el rendimiento y trazabilidad del proceso de consumibles luego de aplicar las medidas expuestas anteriormente. Para este fin, se generaron cinco indicadores:

1. Generación de residuos sólidos: medir el peso total de residuos sólidos:

(Residuos mes actual $(\mathrm{Kg})$

(Residuos mes anterior $(\mathrm{Kg})$

Donde:

- Residuos mes actual = cantidad de residuos generados en kilogramos al mes actual

- Residuos mes anterior $=$ cantidad de residuos generados en kilogramos al mes anterior

Con este indicador se puede medir la variación que hay en el total de residuos sólidos para así determinar los controles pertinentes cuando se presenten incrementos

2. Producción per cápita de residuos sólidos: determinar la cantidad mensual de residuos sólidos ingresados en el área de almacenamiento de residuos al interior del Senado de la República por cada persona:

$\frac{g}{\text { Población }}$

Donde:

- $\mathbf{g}=$ cantidad de residuos ingresados por mes (en gramos)

- Población = cantidad de persona que trabajan en el Senado en el mes

Se espera hacer la medición para que cuando haya tendencia creciente, se puedan generar los controles pertinentes
3. Impacto de mejora en proceso de consumibles: medir el grado de satisfacción de los funcionarios del Senado de la República en relación con las soluciones propuestas en dicha entidad, por cada persona:

(Suma de las puntuaciones positivas $(\mathrm{Kg})$

(Total de valoraciones obtenidas $(\mathrm{Kg})$ * 100

Donde:

- Suma de las puntuaciones positivas = hace referencia a las puntuaciones satisfechas o positivas (mayores a 3 ) en relación con las soluciones propuestas

- Total de valoraciones obtenidas = total de opiniones tomadas

Sirve para conocer la opinión de las personas encargadas de dar vía al proyecto y con esto determinar su viabilidad y para desarrollos de mejoramiento continuo

4. Cumplimiento del pedido:

$$
\frac{\text { Recibidos }}{\text { Pedidos }} * 100
$$

Donde:

- Recibidos $=$ cantidad de productos recibidos

- Pedidos = cantidad de productos pedidos

Es importante tener un control en el cumplimiento de los pedidos para así realizar una adecuada distribución de los productos

5. Calidad:

(No conformes o devueltos)

$$
\text { Pedidos }
$$

Donde:

- No conformes $=$ cantidad de productos defectuosos

- Pedidos = cantidad de productos pedidos 
Se busca determinar la calidad de la recepción de materiales con base en el pedido; es importante tener un control en la calidad de los pedidos recibidos para así brindar una adecuada entrega de los productos. Esto puede complementar el software que se maneja en el Senado para el adecuado control de recibo y entrega de bienes adquiridos y suministros, llamado "Dinámica Gerencial", que está implementado en la Sección de Suministros y en la Unidad de Almacén (la cual maneja inventarios).

Estos indicadores se ejecutan durante y después de aplicar los protocolos diseñados para el proceso de consumibles, son de gran importancia en una organización porque, como lo mencionan Laura Monroy y Neila Simbaqueba, un sistema con este tipo de mediciones proporciona información que apoya la toma de decisiones, y permite lograr un conocimiento mucho más profundo de los procedimientos y su relación con los respectivos responsables [20]. Además, sirven a las empresas como herramienta de mejoramiento continuo para obtener mayor calidad en los productos y servicio.

\section{Análisis de impacto - Matriz}

El análisis de impacto fue realizado por medio de una matriz de Marco Lógico, cuyo inicio consistió en la definición del problema mediante un “árbol de problemas". Según Martelo, Jiménez y Moncaris, esta técnica permite ordenar las ideas presentes en la situación y localizar el problema central o focal que se quiere solucionar, desplegando consigo las causas y efectos de éste [21][22]. Se aplicó esta técnica y posteriormente se realizó un "árbol en positivo" o de objetivos que permitió representar un escenario deseable de la situación actual, convirtiendo el problema principal en la finalidad fundamental [23].

Posteriormente, se generó la matriz, que de acuerdo con Mauricio León y Andrés Paredes, esta técnica se refiere a una tabla que usualmente posee la misma cantidad de filas y columnas (cuatro) en las que se resume un plan; el "Enfoque de Marco Lógico" para la gestión del ciclo de proyectos, se refiere al proceso que comprende las diferentes fases para un proyecto en particular: identificación de problemas, análisis de involucrados, análisis de problemas, análisis de objetivos y análisis de alternativas [24]. Adicionalmente, permite llevar un control sobre los objetivos y las actividades propuestas, con algunos indicadores, medios de verificación (las fuentes de información) y supuestos en su realización.

\section{Discusión}

La estandarización implica analizar detalladamente los procesos de una organización para definir qué elementos establecen las entradas y las salidas para así documentar (lo cual es un paso fundamental) de una forma idónea dichos procesos.

La documentación es uno de los procesos más importantes dentro de una empresa, en Latinoamérica se han realizado diversos estudios asociados al tema, uno de estos fue el realizado por MSc. Onivia Morales Rodríguez, MSc. Roxana González Amador, Dra. C. Hilda Oquendo Ferrer, Dr. C. Néstor Loredo Carballo, Dra. C. Yaíma Filiberto Cabrera y Dr. C. Pablo Galindo Llanes en la Universidad de Camagüey Ignacio Agramonte Loynaz, en Cuba, el cual se tituló "Procedimiento para la documentación de los procesos en los sistemas de gestión de la calidad de la ciencia y la técnica universitaria" [25], en este, se diseñó una estructura documental para un proceso al interior de la universidad, basado en un sistema de gestión de calidad, mediante herramientas similares a las del presente trabajo.

Este estudio se basó en cuatro fases principales, enfocadas en:

1. El establecimiento de las condiciones para el estudio del proceso

2. La caracterización del proceso

3. La definición de elementos de integración del proceso

\section{Documentación del proceso}

Estas fases fueron realizadas con la escogencia de un equipo de trabajo para el proceso, un desglose, detalle y selección de actividades para la caracterización, definición de indicadores y la documentación que tuvo elementos importantes como la elaboración de una ficha del proceso, diagrama

Rev. Ingeniería, Matemáticas y Ciencias de la Información Vol. 8 / Núm. 15 / enero - junio de 2021; pág. 55-66 
del procedimiento y el seguimiento, análisis y mejora de los resultados.

La metodología utilizada en el presente proyecto fue muy similar a la propuesta por los autores de la Universidad de Camagüey, sin embargo, esta última no tiene una estructura tan amplia como la que puede dar una estandarización, la cual tuvo un análisis diagnóstico y procedimental más riguroso, y que por ende, tuvo una documentación que permitió tocar otros aspectos adicionales como los riesgos del proceso, los tiempos, y el detalle de los protocolos desglosados en procedimientos de toda el área.

Como valor agregado y como aporte metodológico, con respecto al proyecto referenciado anteriormente, está la aplicación de herramientas cuantitativas de ingeniería para el diagnóstico de la situación inicial del proceso en la organización, así como la propuesta de tiempos, que, además de medir la duración de cada procedimiento, servirá también como base para otras investigaciones que contengan actividades similares, y que a su vez permita evaluar el impacto del proyecto.

\section{Conclusiones}

Gracias a herramientas de ingeniería como el muestreo estadístico, el diagrama de Pareto, la matriz DOFA, se pueden identificar los puntos más importantes de un proceso y diagnosticar la situación actual del mismo.

La documentación de los procesos contribuye a incrementar la eficiencia en los procedimientos y asegurar su control, promoviendo la calidad y mejora de las actividades.

El trabajo se realizó de forma sistémica con otras áreas asociadas a la Dirección General Administrativa de acuerdo a la alta demanda del proceso que se maneja al interior y en la población flotante de las instalaciones.

La implementación de la estandarización en la estructura del Senado de la República de Colombia permitirá aprovechar las oportunidades de mejora que se evidenciaron en el proceso de consumibles de la División de Bienes y Servicios. Al tenerlo documentado, se podrá llevar un control de los resi- duos, formalidad en los procedimientos, una adecuada utilización de los recursos y la eliminación de actividades que no generan valor, a la misma vez que se contribuye con el ambiente.

\section{Agradecimientos}

Agradecemos particularmente a los funcionarios del Senado, doctoras Silvia Stella Meneses, Gloria Amparo Bustos e ingenieros Jairo Audor y Luis Gabriel Cepeda, por permitir la realización del proyecto y brindar el acceso a las instalaciones, a la información, y por todo el apoyo ofrecido a lo largo del trabajo. También a los expertos que contribuyeron en la estimación de tiempos de los procesos por su disposición y contribución.

\section{REFERENCIAS}

[1] Organización de Naciones Unidas, “Objetivo 12: Garantizar modalidades de consumo y producción sostenibles." Available: https://www.un.org/ sustainabledevelopment/es/sustainableconsumption-production/, 2012.

[2] O. Vargas, E. Alvarado, C. López, and V. Cisneros, "Plan de manejo de residuos sólidos generados en la Universidad Tecnológica de Salamanca," Revista Iberoamericana de Ciencias, vol. 2, no. 5, pp. 83 91, 2015.

[3] A. M. Jiménez Coronado, J. O. Ferreira Simmonds, N. A. G. León Castro, D. E. Martínez Sierra, and J. E. Villarreal Fernández, "Sistema de gestión por procesos para la evaluación del desempeño ambiental a partir de la implementación de diagnósticos PHVA," Producción + Limpia, vol. 11, no. 1, pp. 150-161, 2016.

[4] C. Yunenue, "La estandarización de procesos, como herramienta de mejora a la calidad de procesos administrativos," Universidad Nacional Autónoma de México, 2016.

[5] R. Hernandez Sampieri, Metodologia de la investigación, Mexico: The McGraw-Hill, 2014.

[6] R. A. K. Angarita, “Investigación De Mercados: Un Modelo De Obtención De Estimadores En Variables Cualitativas," pp. 33-40, 2009.

[7] T. Otzen and C. Manterola, "Técnicas de Muestreo sobre una Población a Estudio," International Journal of Morphology, vol. 35, no. 1, pp. 227-232, 2017.

[8] A. Porras Velázquez, "Diplomado en Análisis de información Geoespacial (Tipo de muestreo)," 
Conacyt, p. 14, 2017. https:/ / centrogeo.repositorio institucional.mx/jspui/bitstream/1012/163/1/ 19-Tipos de Muestreo - Diplomado en Análisis de Información Geoespacial.pdf

[9] A. E. Contreras, C. A. Mesías and H. M. A. Rodrgíuez. "Aplicación a beneficiarios de créditos estatales en la ciudad de Bogotá D.C.", 1-20, 2017. https:/ / repository.libertadores.edu.co/bitstream/ handle/11371/1481/contrerasadriana2017. pdf? sequence $=1$ \&isAllowed $=y$

[10] J. M. Izar, "CAPÍTULO IV 4.1 Diagrama de Pareto", Avaliable: https://www.researchgate.net/ publication/303876853_CAPITULO_IV_41_ Diagrama_de_Pareto, 2016.

[11] C. Nikulin and G. Becker. "A Systematic and Creative Methodology to Drive Strategic Management: Chilean. Journal of Technology Management \& Innovatio", 10(1), 1-6, 2015.

[12] C. Guerrero, "Propuesta de un plan estratégico de mercadeo para la empresa El Gran Portal de Mega Eventos", 2016.

[13] G. M. Alonso, "Análisis y Desarrollo Estratégico de una Pequeña Empresa", https://repositorio. upct.es/handle/10317/6121, 2017.

[14] N. A. Leyva and L. T. Ramírez, "Levantamiento de procesos y procedimientos de la empresa Aviles Confecciones S.A.S basado en la NTC 9001:2015," Universidad de Ibagué, Avaliable: https:// repositorio.unibague.edu.co/bitstream/20.500. 12313/1368/1/Trabajo de grado.pdf, 2019.

[15] Instituto Colombiano de Normas Técnicas y Certificación (ICONTEC), «Norma técnica Colombiana NTC-ISO,» Bogotá, 2015.

[16] Instituto Colombiano de Normas Técnica y Certificación, "GTC-45: Guía para la identificación de los peligros y la valoración de los riegos en seguridad y salud ocupacional," Icontec, no. 571, pp. 138, 2010.

[17] N. Alzate and J. Sánchez. “Estudio de métodos y tiempos de la línea de producción de calzado tipo -clásico de dama- en la empresa de calzado Caprichosa para definir un nuevo método de producción y determinar el tiempo estándar de fabricación". Universidad Tecnológica de Pereira, 2014.
[18] G. García and M. Carrillo, “Indicadores de Gestión: Manual Básico de aplicación para Mipymes," no. April 2016, p. 368, 2017.

[19] J. Córdoba and E. Aldunate, "Formulación de programas con la metodología del Marco Lógico". Avaliable: http:/ / www.cepal.org/ilpes/publicaciones/xml/0/43220/SM_N68_Formulacion_ prog_metodologia_ML.pdf, 2011.

[20] L. L. Monroy and N. Simbaqueba, "La importancia de los indicadores de desempeño en la gestión empresarial," Revista Incaing Investigación y Ciencia Aplicada a la Ingeniería, vol. 1, pp. 50-52, 2017.

[21] A. M. Jiménez Coronado, J. O. Ferreira Simmonds, N. A. G. León Castro, D. E. Martínez Sierra, and J. E. Villarreal Fernández, "Sistema de gestión por procesos para la evaluación del desempeño ambiental a partir de la implementación de diagnósticos PHVA," Producción + Limpia, vol. 11, no. 1, pp. 150-161, 2016.

[22] R. J. Martelo, I. Jiménez-Pitre and L. González. Guía metodológica para el mejoramiento del desarrollo de software a través de la aplicación de la técnica Árboles de problemas. Información Tecnológica, 28(3), 87-94. https://doi.org/10.4067/S071807642017000300010, 2017.

[23] S. A. Rios Torres and J. P. Garnica Castaño, "Rediseño Del Proceso Productivo Del Molino Scortum 300 Para Innovaciones Actum S.A.S.," pp. 1-66, Avaliable: https:// repository.eia.edu.co/ handle/11190/2231, 2018.

[24] M. J. León Cárdenas and A. Paredes Murcia, “El marco lógico: Ejemplo de uso en lineamientos para la planificación y la gestión turística municipal en Soacha (Colombia)," Espacios, vol. 40, no. 1, Avaliable: https://www.scopus.com/inward/ record.uri?eid=2-s2.0-85059978861 \&partnerID= $40 \& m d 5=8555 b 009 f 182355 a c 026472$ dbb716c89, 2019.

[25] O. Morales Rodríguez, R. González Amador, D. C. Hilda Oquendo Ferrer, C. Néstor Loredo Carballo, and D. C. Yaíma Filiberto Cabrera C Pablo Galindo Llanes, "Procedimiento para la documentación de los procesos en los sistemas de gestión de la calidad de la ciencia y la técnica universitaria Documentation Procedure for Quality Management of Science and Technology Systems in the University," Dirección, vol. 11, no. 2, pp. 111-135, 2017. 